\title{
Métaphysique des tubes d'Amélie Nothomb: une vie racontée à travers l'intensité linguistique
}

\author{
Métaphysique des tubes by Amélie Nothomb: \\ narrating a life through lexical intensity
}

\author{
Patrizia CRespi \\ Universidad Nacional de Educación a Distancia \\ pcrespi8@alumno.uned.es
}

\begin{abstract}
Reading a book of the belgian writer Amélie Nothomb is an act of passion and dedication: through her characteristic style, filled with rhetorical images that she perfectly masters, the reader plunges into a nice and immoderate atmosphere in which reality merges with exaggeration. This is mostly referred to her autobiographical novels: in Métaphysique des Tubes (The Character of Rain) she travels through times and memories to give us a recollection of her first three years of living. By applying in this book to the point of view of a child, the author let the reader feel the same sensations as the main character, thanks to the accuracy of a precise syntax, where the speech is filled with intensification methods, becoming an implicit network underlying the main history, being by definition the real outline of the description of reality. Intensity is, in Nothomb's book, a stylistic and narrative necessity, revealing its creative force through many lexical forms that we aim to identify and categorise in this article.
\end{abstract}

Key-words

intensity, Nothomb, autofiction.

\section{Resumen}

La lectura de un libro de la autora belga Amélie Nothomb conlleva siempre entrega y pasión. A través de su peculiar estilo, lleno de figuras retóricas aplicadas con gran maestría, el lector se sumerge en una atmósfera agradable y excesiva, donde realidad y exageración se mezclan sin solución de continuidad. Esto es particularmente cierto en sus novelas de inspiración autobiográfica. En Métaphysique des Tubes la autora viaja en el tiempo y en la memoria para devolvernos los recuerdos de sus tres primeros años de vida. Adoptando el punto de vista de cuando era niña, Nothomb consigue que sus lectores compartan las sensaciones de la protagonista a través de una sintaxis extremadamente cuidada y de un discurso colmado de procedimientos de intensificación; una verdadera red subyacente a la trama principal que asume, mediante las múltiples expresiones léxicas de la intensidad, la función de estructura de soporte de la novela en su descripción de la realidad.

\section{Palabras clave}

intensidad, Nothomb, autoficción. 


\section{Introduction}

Amélie Nothomb naît à Kobe (Japon) en 1967. Après avoir voyagé sans cesse dans les différents pays d'affectation de son père, Consul de la Belgique en mission en Asie ainsi qu'en Amérique du Nord, elle a commencé son activité d'écrivaine à Bruxelles à l'âge de 17 ans, lors de sa première année d'études universitaires.

Nothomb a créé un personnage littéraire assez bizarre, devenu le protagoniste de la plupart de ses récits écrits à la première personne; un personnage qui lui ressemble, dont on connaît la famille (identique à celle de l'auteure) et qui vit des expériences que nous savons avoir été vécues par Nothomb dans sa vie réelle. S'agit-il de la même personne? Oui et non. Il y a une coïncidence physique, bien sûr, entre l'écrivaine et son alter ego belgo-japonais, mais la construction identitaire de la première passe souvent par l'affirmation et le développement du second.

Métaphysique des Tubes est le onzième livre publié par Amélie Nothomb ${ }^{1}$, où elle voyage dans le temps et dans la mémoire pour nous livrer les souvenirs de ses trois premières années de vie. L'enjeu est fondamental, puisque, d'après elle: "la naissance de tout être humain est une véritable genèse". Il s'agit donc d'un moment de sa vie qui mérite d'être approché avec un style sérieux, limpide, et toujours très vif, afin de dessiner dans les moindres détails l'émotion correspondante à ce big bang personnel.

En choisissant pour ce récit le point de vue de l'enfant, Nothomb réussit à nous faire partager les sensations intenses ressenties par la protagoniste, en nous emportant sur le bateau de la justesse syntaxique, où les procédés de l'intensification remplissent le discours en se posant comme un réseau sous-jacent à l'histoire racontée, et qui devient par sa propre définition la véritable structure portante de la réalité décrite.

À propos de l'écriture de l'enfance, Michel Zumkir dans son livre Amélie Nothomb de $A$ à $Z$ nous offre une intéressante déclaration de l'auteure, qui approfondit son attitude par rapport au langage qu'elle a choisi d'employer dans Métaphysique des Tubes. Ces explications nous rapprochent de son choix linguistique, derrière lequel il y a une claire volonté de création et de contrôle de la vraisemblance.

Quand on écrit sur la toute petite enfance, on a un choix. Soit on décide de le faire avec
le langage qui était le sien à l'époque, mais cela est impossible, car j'ignore comment
je parlais quand j'étais toute petite. Et si j'essayais de reproduire ce langage-là, je fe-
rais quelque chose de tout à fait stupide... Il y a un autre pari qui consiste à prendre
le langage d'une personne de trente-trois ans, et même s'il y a un écart, qu'un enfant
n'utilisera pas les mots que j'emploie, essayer de traduire ce qu'était ma pensée à ce
moment de ma vie. Franchement, je ne me souviens pas des évènements avant deux ans

1 Il est important de souligner qu'il s'agit de son onzième livre publié, car Nothomb, qui se définit comme une graphomane, écrit tous les jours de sa vie depuis ses 17 ans, pour une moyenne de trois à quatre livres rédigés par an. En début d'année, elle en choisit un - suivant des critères que nous ne sommes pas censés examiner ici - qui sera offert à son éditeur et publié lors de la rentrée scolaire, chaque année au mois d'août. 
et demi, mais j'en conserve de vagues impressions. Si je descends très loin en moi, je peux les retrouver. C'est là que se situe mon pari ${ }^{2}$.

À aucun moment, dans ce texte, l'auteure ne révèle le prénom de la protagoniste. Elle l'appelle Dieu, le tube ou la Plante, ou encore tout simplement moi; la seule évocation du prénom étant le passage où l'enfant de deux ans et demi éprouve une communion idéale avec l'eau'.

Il s'agit d'un choix évident, puisque les allusions aux prénoms des autres membres de sa famille ainsi qu'aux personnes qui apparaissent dans son monde sont nombreuses dans le texte. La volonté de ne pas partager un identifieur socialement reconnu tel que le prénom est étroitement liée au jeu identitaire qui situe son témoignage littéraire à la frontière entre l'autobiographie et l'autofiction. Le je nothombien reste mystérieux et ouvert, puisqu'il se construit de façon sinueuse non seulement à travers les souvenirs autobiographiques, mais aussi dans le labyrinthe de la création fictionnelle.

L'interrogation sur la construction de l'identité chez Nothomb a fait l'objet de nombreuses analyses, se révélant fondamentale dans l'approche des thèmes abordés dans sa production littéraire ${ }^{4}$ Christine Ferreira Azzi ${ }^{5}$ approfondit le rapport qui s'établit entre le discours autobiographique et le discours fictionnel nothombiens, tout en le comparant au lien qui unit la mémoire et l'écriture de l'écrivaine. Dans son Mémoire intitulé L'évolution physique et mentale de la protagoniste dans cinq romans d'Amélie Nothomb ${ }^{6}$, Laila Boubli identifie le langage comme la véritable réponse que Nothomb a trouvée aux débordements de ses sentiments. Elle suit le développement intime de l'écrivaine par le chemin de l'évolution de la protagoniste de ces romans autobiographiques. María Luisa de la Oliva ${ }^{7}$, aboutit à l'identification de la blessure/séparation qui a donné naissance à Nothomb, dans son étude sur la place qu'occupe le je nothombien dans l'espace créé grâce à son "écriture-tissu" (De la Oliva 2012: 142). Cette blessure s'avère incurable et témoigne d'une véritable "psychose infantile" (De la Oliva 2012: 143) dans le texte qui nous occupe.

Tout en tenant compte de ces approches intéressantes, nous avons décidé de centrer notre étude sur l'analyse de l'expression de l'intensité qui caractérise la vision extrême que l'auteure projette sur la vie; dès lors, nous n'approfondirons pas cette interrogation identitaire. Nous découvrirons, par contre, comment ce trait linguistique n'est pas loin d'être également un trait fondateur de l'identité de l'auteure. Amélie Nothomb est une écrivaine très culte qui, étant profondément consciente de leur importance, maîtrise la syntaxe et le lexique

2 Citée par Michel Zumkir.

3 Cf. page 11 de notre texte.

4 Voyons la description très juste qu'Henri Delangue nous offre: "Le je nothombien reste tel que son auteur: mystérieux, énigmatique et dont l'identité semble infinie." (Delangue, 2014: 140).

5 "Je est un autre: Amélie Nothomb e a escrita autobiográfica."

62011.

7 “L'en-je lacanien" (2012). 
en les employant de façon très exacte pour construire une réalité sémantique et narrative se nourrissant de cette approche intense qui idéalise et magnifie les émotions de l'auteure. Un examen de la fonction qu'assume l'intensification linguistique dans l'économie sémantique de ses textes lui accorde l'étendue que l'auteure a maintes fois affirmé rechercher par ses choix stylistiques.

Dans le texte que nous analysons ici, l'écrivaine Amélie a pour but lexical de traduire par les mots sa sensibilité aiguë et sa capacité de s'émerveiller comme un enfant, et ceci, par l'emploi d'un style excessif où le personnage Amélie est le plus souvent pris dans un vortex émotionnel. "Mon pari était de saisir des choses qui étaient de l'ordre de l'impression. Je pense que l'écriture est un moyen d'investigation tellement profond que rien qu'en écrivant, on peut retrouver des impressions de cet âge tubulaire [...]"8. Elle ne saurait ni vivre ni écrire sans l'accompagnement presque musical ${ }^{9}$ de ces superlatifs, puisque c'est justement avec une telle approche qu'elle saisit la vie: de façon superlative.

\section{Corpus}

Le langage et son emploi aussi bien à l'oral qu'à l'écrit assument un rôle fondamental dans toute la production littéraire nothombienne. Elle a affirmé maintes fois que les mots, à travers la littérature, lui ont offert un endroit stable de référence, dans une vie caractérisée par de nombreux déplacements et des abandons douloureux.

Afin de réaliser une analyse linguistique de l'œuvre de Nothomb, nous avons considéré intéressant d'examiner un texte où le langage est mis au centre du récit. Métaphysique des tubes est en ce sens le livre parfait. L'auteure y expose sa propre découverte du langage, non seulement par rapport à l'oralité, mais aussi quant au pouvoir de la parole écrite, produit d'une profonde réflexion et d'un choix raisonné, et donc encore plus perçante.

Lorsqu'elle écrit, Nothomb recherche une introspection qui l'amène à retrouver ses expériences intimes, afin de savoir les narrer de façon exacte dans ses textes autobiographiques, autant que pouvoir les relancer sur les personnages qui peuplent ses romans de fiction. Dans Métaphysique des tubes, elle affiche son talent pour récupérer les sensations de ses premières années, mais aussi pour remplir avec l'imagination les éventuels trous de mémoire.

Dans ce jeu de miroirs de la création qui caractérise le texte que nous avons choisi, l'auteure peut déployer ses facultés de démiurge: elle crée une réalité dans l'histoire, et ce faisant elle permet aux lecteurs de participer au spectacle inédit de l'assomption de la proposition créatrice linguistique de la part d'un enfant, comme le fait remarquer Amanieux: "Elle cherche à découvrir le langage, qui seul réunit pouvoir, savoir et vouloir. Métaphysique des tubes se présente comme une aventure formatrice pour le maîtriser." (Amanieux 2005: 30)

8 "La syntaxe c'est l'harmonie. [...] La syntaxe, c'est le solfège" (Serrell, 2017: 109).

9 “La syntaxe c'est l'harmonie. [...] La syntaxe, c'est le solfège" (Serrell, 2017: 109). 
L'histoire, qui se déroule à Kobe, ville de la région japonaise du Kansaï, commence par la Genèse particulière d'une auteure qui se compare à la fois à Dieu (avec majuscule, en plus) et à un tube, dont la fonction digestive était la seule importante. La petite Amélie, qui a une sœur et un frère ainés, est une enfant apathique, que ses parents appellent gentiment "la Plante" à cause de son manque absolu d'activité. Ils essayent de la réveiller par mille approches différentes, mais rien n'y fait: le récit nous révèle qu'à deux ans, Dieu n'avait toujours pas fait le moindre mouvement. Un jour, sans raison, elle se met à hurler et n'arrête plus, elle semble être toujours en colère, ce qui fait regretter sa situation de Plante. La grandemère paternelle, prévenue du changement miraculeux, arrive de Belgique pour rendre visite à la famille et provoque le réveil de l'enfant, tel une deuxième naissance, grâce à un bâton de chocolat blanc. Du point de vue syntaxique, c'est à cet instant que le style passe d'un narrateur externe omniscient qui s'exprime à la troisième personne du singulier, à un narrateur qui s'identifie à la protagoniste et narre les évènements à la première personne du singulier. Amélie rattrape le temps perdu et devient une enfant sage et éveillée. Son premier mot est maman, suivi de papa. Elle nous raconte sa relation d'affection et de respect pour Nishio-San, douce gouvernante japonaise qui l'adore. Un jour, Amélie apprend le décès de sa grandmère, nouvelle qui lui provoque de profondes réflexions pseudo-philosophiques. Peu après, une deuxième gouvernante s'ajoute à la famille, Kashima-San. La relation entre Amélie et cette dernière s'avère être à l'opposé de l'amour qui la lie à Nishio-San, et empire de jour en jour. Quand la famille se rend à la mer, l'enfant, qui aime beaucoup nager et être dans l'eau, faillit se noyer en perdant pied. C'est un ami de son frère qui prévient la mère et la sauve. Le mois de mai arrive: Amélie découvre les carpes, poissons symbolisant au Japon les garçons, qu'elle haït immédiatement. Un jour, elle écoute une conversation privée entre Nishio-san et Kashima-San, qui - par ses moqueries et critiques à la famille belge qui l'accueille - suscite chez sa collègue la décision de démissionner. Quand Amélie apprend de sa mère qu'un jour ils devront quitter le Japon, elle sent le monde s'effondrer autour d'elle; heureusement, $\mathrm{Ni}$ shio-San décide de rester avec eux. C'est le jour du troisième anniversaire de la protagoniste: elle désire intensément un éléphant en peluche, mais ses parents se trompent complètement en lui offrant trois carpes détestées. Elle les appelle Jésus, Marie et Joseph. Obligée de les nourrir, un jour elle se sent à tel point dégoûtée qu'elle se laisse tomber dans le bassin, où sa tête heurte un rocher. Kashima-San la voit, mais ne prévient personne; heureusement, $\mathrm{Ni}$ shio-San est présente: elle la sauve et l'emmène avec Mme. Nothomb à l'hôpital, où la petite est rapidement soignée. Le récit se clos sur une interrogation: cette tentative de suicide s'estelle réellement passée? Une cicatrice sur sa tempe gauche laisse l'écrivaine et ses lecteurs pareillement perplexes.

Nous nous sommes également appuyé sur un autre livre de Nothomb auquel nous ferons référence à plusieurs occasions: Hygiène de l'assassin, son premier livre publié (1992). Il faut relever que, d'après Nothomb, elle serait à chercher plus dans ses romans de fiction 
que dans ses œuvres autobiographiques, car dans ceux-là elle se sent beaucoup plus libre, car elle s'exprime à travers ses personnages.

Dans Hygiène de l'assassin, conçu quasi comme une pièce théâtrale où la suprématie du dialogue est évidente, nous retrouvons de nombreuses considérations sur la langue et la littérature, sous forme de propos tenus par son protagoniste -un vieil écrivain proche de sa fin-, que plusieurs critiques ont identifié comme un alter-égo de l'auteure. Zumkir (2007: 138) n'a aucun doute à ce sujet: "Prétextat Tach est le double de l'écrivaine, celui qui porte ses idées sur la littérature à leur paroxysme."

L'importance de ce livre pour notre analyse de l'intensité réside principalement dans la présence constante de nombreux propos linguistiques et stylistiques prononcés par son protagoniste, qui en font presque un manuel de théorisation de l'art littéraire.

\section{Approche et méthodologie}

Le concept de degré d'intensité, intensification, est une notion ancienne, qui a déjà fait l'objet de nombreuses études et analyses linguistiques. Pour une définition abstraite de cette idée, nous nous rapprocherons de la représentation proposée dans Romero (2007: 56), qui décrit l'intensité comme une tension entre deux états: celui qui peut être considéré normal nous l'appellerons état de référence -et celui que l'objet de l'intensité atteint, à la suite du procédé intensifieur- l'état intensifié. C'est une représentation efficace pour notre analyse, dont l'un des objectifs est de démontrer qu'à travers un langage excessif et intense, l'auteure réussit à créer une réalité autre, c'est-à-dire un état différent du normal.

Le degré d'intensité du contraste entre état de référence et état intensifié peut être exprimé dans un texte écrit à l'aide de plusieurs variantes, parmi lesquelles les adjectifs superlatifs, les adverbes et les locutions ne sont pas les plus difficiles à repérer; les moins évidents à une première lecture étant les sémèmes dont le sens même marque l'intensification du discours (Do Nascimento 2008: 80).

Lorsque l'expression intense mène à une opposition explicite entre les deux états, ceci peut servir comme point de départ pour que notre auteure joue avec les possibilités lexicales en explorant jusqu'à quel point elle peut pousser le degré d'intensité sans pour cela sortir du véridique. C'est un procédé typique chez Nothomb qui se déroule selon les deux catégories d'expression du contraste: dans les figures d'opposition, telles que l'antithèse, le paradoxe ou l'oxymore, où les éléments qui s'opposent apparaissent en même temps; et dans les constructions comme la focalisation, l'ellipse et l'ironie, qui ressortissent plutôt d'un contraste où l'écrivaine met en évidence un élément absent dont la découverte n'est possible que grâce à l'implication du lecteur dans la décodification du message.

Dans notre étude de l'intensification nous partons du concept de fonction lexicale, qui a été créée par Igor Mel'čuk à la fin du XXème siècle, dans le cadre de la Théorie Sens- 
Texte et reprise pour son application pratique par Alain Polguère ${ }^{10}$. Il s'agit d'un concept qui, désormais, fait partie de la terminologie courante dans grand nombre d'approches des différentes branches de la linguistique: lexicographie, lexicologie et sémantique entre autres. Il occupe une place fondamentale aussi bien dans l'enseignement de FLE que dans l'apprentissage des connaissances lexicales de base en français langue maternelle. Le système des fonctions lexicales (dorénavant: FL) modélise de façon très efficace les liens sémantiques entre les unités lexicales qui forment une langue. C'est un instrument de décodification linguistique fiable qui permet la compréhension autant du style lexical d'un texte que de ses implications sémantiques relatives aux choix personnels de son auteur

La FL Magn(um) rentre dans la catégorie des fonctions lexicales standard, étant donné qu'elle exprime une caractéristique de base du langage commune à toutes les langues, à savoir: l'expression de l'intensification. La FL Magn associe à une Lexie toutes les lexies ${ }^{11}$ dont le but est d'appliquer le sens générique d'intensification, c'est-à-dire que la FL Magn peut mettre l'accent sur l'une ou l'autre composante sémantique de la lexie qu'elle modifie, mais elle a toujours pour effet une lexie à un état intensifié, tel que nous l'avons défini auparavant.

Voyons un exemple de cette propriété, tiré de notre corpus: "Il était satiété et éternité." (Nothomb, 2000: 6). C'est une phrase définitoire par laquelle l'auteure qualifie Dieu, où celui-ci est l'enfant Amélie pendant ses premiers mois de vie, avant la découverte de la parole et la "re-naissance" de la main du chocolat blanc offert par la grand-mère paternelle. Ici, la FL Magn est exprimée de façon extraordinaire par deux substantifs, dont la valeur sémantique correspond à 'intense': satiété pour l'intensité qualitative qui renvoie à un état de tranquillité et de plénitude (substantif qui apparaît plus tôt dans le texte, en rapport avec la vie); éternité pour l'intensité de la durée, qui, par définition, peut être appliquée avec exactitude uniquement au concept de Dieu, puisqu'elle est une des propriétés inhérentes à la divinité.

À partir de cette grille d'analyse fondée sur la fonction lexicale, nous avons procédé à identifier les expressions de l'intensité dans Métaphysique des tubes, et à en dresser une hiérarchie, afin de définir les traits linguistiques fondamentaux du style nothombien. Cette tâche s'est avérée assez compliquée, puisque le mélange est très fluide et le texte entier dans sa complexité exprime l'intensité presque sans solution de continuité. Évidemment, la référence à la hiérarchie ne tient pas compte de la fréquence d'application d'une expression, mais elle relève plutôt de son influence sur la signification globale du texte et de son importance dans l'économie sémantique du récit.

Les évidences soulevées par ce procédé nous amènent à considérer que la syntaxe nothombienne, remplie d'expressions de l'intensité, est capable de donner naissance à une réalité pleine et véridique, s'inscrivant de droit dans le cadre de l'autofiction.

10 Pour une étude plus approfondie des fonctions lexicales et de leur modélisation, $c f$. Polguère (2002).

11 La définition de Lexie peut être relative à un lexème (lorsqu'elle est constituée par un seul élément lexical) mais aussi à un phrasème (expression contenant plusieurs éléments, soit-elle libre, semi-figée ou figée). 


\section{Autofiction vs autobiographie}

Métaphysique des tubes est écrit dans sa majorité à la première personne du singulier, mais il n'est pas, pour autant, un récit exclusivement autobiographique, au sens traditionnel du terme, c'est-à-dire: où le principe fondateur serait celui d'un regard introspectif et subjectif, qui opérerait forcément une sélection des souvenirs et, par conséquent, des évènements mis en scène.

Nothomb forge le concept du regard dès les premières pages du livre, là où il est question de mettre le lecteur au courant de ce qu'il va trouver par la suite: cette notion semble donc mériter un bref approfondissement.

"La vie commence là où commence le regard" (Nothomb, 2000: 6), nous explique-telle avec conviction et un propos narratologique clair: le personnage de son livre est, au début, l'objet d'un regard qui n'est pas encore le sien, jusqu'à ce qu'il s'attribue cette propriété, en déplaçant le niveau du récit d'un narrateur externe et omniscient à celui de la coïncidence entre personnage et narrateur. Est-ce que cette transition serait possible dans une vraie autobiographie?

Le double jeu que l'écrivaine propose à ses lecteurs devient passionnant: faut-il accepter tout ce qu'on va lire comme une spéculation fictionnelle visant à nous entretenir du sujet de ses aventures, sur la base de quelques souvenirs pourtant véridiques? Ou bien, la vérité est-elle à chercher dans le récit même, dans la figure que ces mêmes souvenirs assument pour nous atteindre, c'est-à-dire dans le langage et le style? Au fond, c'est partie remise pour le lecteur: la réponse importe peu dans la vision d'Amélie Nothomb, puisque l'écriture représente pour elle un plaisir intime, indépendant de l'acceptation d'autrui. Il se peut que, grâce à cette recherche du plaisir pour lui-même, elle professe une liberté totale d'écriture par rapport aux contraintes des ventes ou de la critique littéraire: “Quand je vous disais qu'on me lisait sans me lire! Je peux me permettre d'écrire les vérités les plus risquées, on n’y verra jamais que des métaphores." (Nothomb, 1992: 141).

Cette liberté fondamentale se reflète aussi dans la tendance hyperbolique à l'intensification du style, et n'est que la suite logique de la découverte qu'elle avait faite étant petite, lorsqu'elle jouait à mentir à sa sœur et que la méfiance de l'aînée la poussait à perfectionner de plus en plus ses histoires imaginaires, dans une "quête de crédibilité" (Nothomb, 2000: 114) vouée à l'échec. La prise de conscience de cette situation a déclenché la vocation littéraire de la petite en la dirigeant, future écrivaine en herbe, sur la route de l'autofiction (dans ce cas, nous assumons le sens littéral d'inventer des fictions pour soi-même ${ }^{12}$ ), où la seule chose importante est que les histoires soient crédibles dans sa tête.

Comment alors considérer ce texte, si on devait le cataloguer sous l'aspect de l'identité du narrateur, à la vue de toutes les considérations faites jusqu'ici? Pourquoi est-il incorrect

12 La décision est prise: "Je continuerais à inventer, pour mon plaisir" (Nothomb, 2000: 115). 
de le classifier comme autobiographie? Nous allons essayer de justifier la définition du livre comme une œuvre d'autofiction, ce genre hybride où Nothomb serait héritière d'une tradition qui remonte à Marcel Proust, qu'elle affirme adorer, en passant plus récemment par le Prix Nobel français Patrick Modiano.

Dans le but de cette démarche définitoire, il nous semble nécessaire de commencer par tracer un tableau d'ensemble, en guise d'introduction à la technique de l'autofiction, qui nous intéresse de près, car elle caractérise Métaphysique des tubes, ainsi qu'une grande partie de la production nothombienne.

Les études critiques littéraires reconnaissent trois types de texte écrits à la première personne du singulier: l'autobiographie, l'autofiction, ou encore le roman autobiographique - où l'auteur utilise certains épisodes réels de sa vie, mais en se cachant derrière des personnages inventés. Pour revenir à Métaphysique des tubes, nous partageons la définition proposée par Henri Delangue, qui parle plutôt de "récits de vie" (Delangue, 2014: 132) où la vie réelle voyage à côté de celle qui nous est racontée. L'association des deux étant libre, elle permet à l'écrivain le remaniement des souvenirs et l'ajout de l'imagination, là où ces premiers ne suffiraient pas ou ne coïncideraient pas avec la tonalité littéraire souhaitée par l'auteur. Par rapport à la chronique et au développement temporel de l'œuvre, nous remarquerons que dans tous ses romans personnels, "Nothomb répond à l'une des règles fondamentales de l'autobiographie, c'est-à-dire que la chronologie des faits est respectée et logique." (Delangue, 2014: 132): ceci est valable aussi pour le texte analysé. Pourquoi alors nous affirmons qu'il s'agit d'autofiction et non d'autobiographie? Afin de bien comprendre notre affirmation, voyons comment le cadre théorique nous aide à délimiter les caractéristiques de l'autobiographie. C'est un genre très connu et adopté par les écrivains depuis l'âge classique; de plus, le lecteur contemporain a très facilement accès à des œuvres autobiographiques, vu que depuis le siècle passé il n'y a pas que les lettrés qui s'adonnent au récit de leur vie, mais aussi les sportifs, les artistes et les célébrités de tout genre.

Pour une définition stylistique de l'autobiographie, remettons-nous à celle du critique Philippe Lejeune (2005: 15): “l'autobiographie (récit racontant la vie de l'auteur) suppose qu'il y ait identité de nom entre l'auteur (tel qu'il figure, par son nom, sur la couverture), le narrateur du récit et le personnage dont on parle." L'autobiographie est rédigée d'habitude à la première personne, c'est-à-dire que l'identité du narrateur coïncide avec celle du protagoniste de l'histoire racontée, ainsi qu'avec l'auteur qui se trouve hors-texte. Voilà une première différence par rapport à Métaphysique des tubes: nous avons remarqué dans cette étude que jamais dans le texte nothombien n'apparait le prénom de la protagoniste.

Nous avons affirmé auparavant que le texte qui nous concerne est une œuvre autobiographique sui generis; voyons donc à quoi faisons-nous référence par cette définition. L'autofiction est à la fois un concept et un néologisme, qui furent inventés en 1977 par 
Serge Doubrovsky dans le livre intitulé: Fils, et repris plus tard par Vincent Colonna pour en donner une définition assez complète: il affirme dans son Mémoire que ce terme indiquerait une “œuvre littéraire par laquelle un écrivain s'invente une personnalité et une existence, tout en conservant son identité réelle (son véritable nom)" (Colonna, 1989: 30); dans la description autofictionnelle, nous aurons donc affaire à une véritable ré-invention du soi consciente de la part de l'auteur, une espèce d'autobiographie romancée qui devienne le trait d'union entre la fiction et la biographie pure. Le même Doubrovsky, critique universitaire français et professeur aux États-Unis, était également auteur de littérature, ayant écrit plus d'une œuvre d'inspiration autofictionnelle (à partir de Fils, déjà cité ci-dessus). Depuis, le style s'est répandu parmi les écrivains contemporains et le terme a connu une forte diffusion, ce qui pourrait amener à des confusions d'ordre descriptif. Il faut dire que Doubrovsky éprouvait une forte aversion pour l'autobiographie classique, qu'il tachait de mensonge; il détestait le style narratif, qu'il appelait de façon dénigrante "la belle forme" et contre lequel il appliquait dans ses textes une liberté totale du langage, dans un certain sens très proche de l'écriture automatique des surréalistes. L'autofiction telle qu'il l'exprime, devient une forme plus démocratique d'autobiographie, dans le sens où, ne visant pas à créer des vies de légende, elle ne met pas en place des recours stylistiques grandiloquents, mais propose plutôt un contenu réel raconté par un langage vrai. Sur ce point, plusieurs commentaires seraient possibles par rapport à ce que l'on considère un langage vrai et un contenu réel, mais la définition de ces attributs n'est pas le but de notre étude. D'après la vision de Doubrovsky, l'autofiction serait donc un reflet de la vérité, mais pas nécessairement de la réalité. Cette différence est particulièrement importante par rapport à l'écriture nothombienne et notamment au texte qui nous intéresse.

Dans Métaphysique des Tubes, tout ce que l'auteure raconte est vrai, puisqu'il s'agit surtout de sentiments vécus, de sensations intimes, d'émotions provoquées par les premières découvertes dans la vie d'un enfant. Cependant, tout ne peut pas être réel, car même une mémoire prodigieuse comme celle de Nothomb peut avoir des failles, surtout s'il s'agit de remémorer des évènements de la toute petite enfance; mais le lecteur en est conscient et l'accepte volontairement. Il n'est pas vraiment question, dans ce livre, de nous révéler les détails de la vie japonaise de la petite Amélie et des personnes qui l'entouraient, mais plutôt de nous offrir, avec toute la sincérité possible, la vision d'une enfant qui vit une situation particulièrement peu courante, et qui l'apprivoise à fond grâce à une sensibilité extraordinaire.

C'est à ce moment que nous identifions l'importance du lexique de l'intensité comme expression directe d'une réalité intime qui - se superposant à la réalité vraie - dessine une couche riche en émotions dans laquelle on retrouve l'identité de l'auteure.

Le passage qui choque le plus le lecteur par rapport à la véracité de l'histoire est probablement celui de la tentative de suicide dans l'étang aux carpes (Nothomb, 2000: 146); même si, d'après Jacqueline Harpman, amie de Nothomb, psychanalyste et aussi écrivaine, 
il n'y a pas de doutes: “tout dans le roman prouve que c'est vrai." (Zumkir, 2007: 83). Le fabuleux monde d'Amélie n'est pas moins vrai s'il n'est pas réel. Elle en est consciente et pour cela elle s'amuse à créer cette réalité avec les mots, sans que le problème de la vraisemblance ne la touche: “Au fond, cela m'était égal, qu'on me croie ou non.” (Amanieux, 2005: 73).

Là où le récit autobiographique traditionnel opère une sélection dans la mémoire et manifeste une dangereuse tendance à organiser les souvenirs dans un discours linéaire suivant sans solution de continuité le temps réel, l'autofiction renonce à la censure et atteint sans coupures le récit de la vie telle quelle, visant à nous faire part de la puissance de ses sentiments. Dans ce niveau d'idées, mis à part le côté autobiographique relatif aux faits purs et simples, nous avons examiné comment le procédé linguistique de l'intensification occupe une place fondamentale dans le texte et assume une valeur de contexte personnel descriptif ${ }^{13}$.

L'écriture de Nothomb se caractérise, en ce qui concerne à la position qu'elle assume dans la relation avec ses lecteurs, par le fait qu'elle ne donne pas de paratexte relatif à ses œuvres, elle n'ajoute pas d'explications sous forme de prologue ni d'épilogue, mais elle aime jouer avec le récepteur du message. Voyons par exemple, dans Métaphysique des tubes, l'ajout du mot roman sous le titre: un choix tout à fait étrange, qui augmente la sensation de confusion et de doute par rapport à la véridicité de ce que nous allons lire. Cette définition nous fait penser que Nothomb souhaiterait s'éloigner définitivement du concept d'autobiographie. En revanche, sur la quatrième de couverture de l'édition Livre de Poche, une synopsis anonyme annonce explicitement une "autobiographie de zéro à trois ans" tout en insistant sur le fait que l'auteure nous "révèle des aspects ignorés de sa personnalité". La confusion est accrue par quelques détails que nous retrouvons dans le même passage et qui ont attiré notre attention: d'abord, l'écrivaine est définie comme "romancière" ayant obtenu le Grand Prix du Roman (encore ce mot); ensuite, la première critique, signée par Thierry Gandillot ${ }^{14}$, provoque un véritable tourbillon de définitions possibles: "Amélie a trois ans, sa vie est un roman”. En quelques mots, voilà ce qui pourrait nous donner la clef pour envisager un classement de cette œuvre dans la catégorie d'autofiction. Le mélange continue dans la critique suivante ${ }^{15}$, stimulé par la référence littéraire à Marcel Proust qui, nous l'avons dit plus haut, est un des pères de l'autofiction ${ }^{16}$, ainsi qu'un auteur vénéré par Amélie. Le rappel à la Comtesse de Ségur, à qui on attribue traditionnellement la citation: "N'écris que ce que tu as vu", ne peut que creuser davantage le gouffre dans lequel on nous jette, en proie aux doutes et aux questions identitaires, dans un rébus narratif qui ne se résoudra probablement qu'une fois que

13 "[...] je ne suis pas là pour écrire la vérité, je suis là pour écrire ma vérité" (Amanieux, 2005: 73).

14 La citation est extraite de l'article intitulé "Nô, carpes et chocolat", disponible en ligne sur le site $<$ https://www. lexpress.fr/culture/livre/metaphysique-des-tubes_797228.html>

15 La deuxième "Grâce à une étonnante économie de vocabulaire, le style de Mademoiselle Nothomb sert à la perfection les bonheurset malheurs d'Amélie que l'on croirait pensés par une comtesse de Ségur qui aurait fauté avec Marcel Proust".

16 "Le plus fameux exemple de ce type, est sans doute de Proust", affirmation de Durya Dusanic dans 1'article intitulé "La fictionnalité de l'autofiction", disponible en ligne sur le site<http://www.autofiction.org/index. php?post/2012/10/27/La-fictionnalite-de-lautofiction>. 
MÉTAPHYSIQUE DES TUBES D'AMÉLIE NOTHOMB: UNE VIE RACONTÉE À TRAVERS L'INTENSITÉ...

la production littéraire nothombienne aura atteint le statut de géoglyphe (Serrell, 2017: 113), et qu'on aura la possibilité de la regarder de haut comme les dieux, en appréciant le dessin de son histoire dans sa complexe simplicité.

\section{Description et application de l'intensité dans Métaphysique des tubes}

Le style nothombien est en apparence facilement reconnaissable: ses romans, qu'ils soient de fiction pure ou d'autofiction, descriptifs ou dialogués à la limite de la pièce de théâtre, se caractérisent tous par leur simplicité et immédiateté, ainsi que par la rigueur de la grammaire et l'audace dans la créativité lexicale. Cependant, plusieurs niveaux de lecture sont possibles pour ses œuvres, parmi lesquels l'approche linguistique est une des plus passionnantes, compte tenu de l'importance que Nothomb octroie à son univers syntaxique.

Bien que cela puisse paraître contraire au sujet de notre étude (la construction de l'intensité), le trait fondamental de l'écriture nothombienne est sans aucun doute la simplicité, une simplicité voulue et minutieusement construite, qui étonne le lecteur par la densité de son expression.

L'écrivaine belge applique à l'écriture son propre mantra: "Pourquoi faire compliqué, quand on peut faire simple?" (Nothomb, 2000: 38). Conscients de la grande admiration qu'elle professe pour la littérature classique ${ }^{17}$, qu'elle connaît et étudie avec ferveur, ce propos nous fait penser à un texte du Siècle d'Or espagnol, écrit par Baltasar Gracián et intitulé Oráculo manual y arte de prudencia; un recueil de trois cents maximes et aphorismes commentés, publié en 1647 comme guide de conduite pour l'homme prudent. Le conseil exprimé par le moine espagnol dans le vingt-septième aphorisme de ce recueil didactique coïncide avec la recherche nothombienne d'un style simple, mais concentré: "Lo bueno, si breve, dos veces bueno". Le livre de Gracián était aussi l'un des plus aimés par le philosophe Nietzsche ${ }^{18}$, pour qui Amélie Nothomb professe une véritable passion, à tel point qu'elle le considère le seul auteur qui lui ait vraiment sauvé la vie (Amanieux, 2005: 244). Voici un lien de style qui s'étire à travers les siècles, en attachant ces trois auteurs du fil rouge de la densité expressive.

Nous retrouvons chez elle une admirable simplicité, entendue donc non pas comme réduction et élimination, mais plutôt comme intensification, obtenue grâce à plusieurs procédés tels que le choix minutieux des adjectifs ou la construction hyperbolique presque paradoxale, ainsi que l'opposition et surtout l'ironie, que nous identifions comme l'autre trait fondamental de son écriture narrative. L'ironie nothombienne a fait l'objet de plusieurs

17 Sur ce sujet, nous renvoyons à l'affirmation catégorique de son alter ego Prétextat Tach dans Hygiène de l'assassin: "Le classicisme ne commet jamais de faute de goût." (Nothomb 1992: 200).

18 Nietszche aurait affirmé, à propos du recueil de l'auteur espagnol: “L'Europe n'a produit rien de plus fin et compliqué en matière de subtilité morale" (cité dans: Lázaro y Tusón, 1990: 135). 
études ${ }^{19}$ et mérite un approfondissement raisonné et indépendant de cette analyse de l'intensité linguistique.

Interrogée sur le sujet de l'apparente simplicité de son style, elle nous a expliqué que “(s)on langage relève toujours de la litote ${ }^{20}$ ", et que ce résultat n'est atteint qu'à base de se réfréner, de s'imposer des expressions capables de condenser dans la moindre quantité possible de signifiants la plus grande amplitude de signifiés (Amanieux, 2005: 247).

Les livres autofictionnels de Nothomb nous la présentent comme quelqu'un de très sensible, pour qui les émotions sont de véritables monuments qui méritent d'être exprimés de façon sublime. Il est donc nécessaire chez elle de réfléchir avec attention à chaque syntagme avant de le mettre noir sur blanc, puisqu'il faut que l'écriture corresponde au souvenir, qu'elle soit capable de reconstruire une réalité qui a été vécue à 360 , où les moindres détails émotionnels sont parcourus jusqu'à la lie. Évidemment, dans une poétique pareille, chaque construction syntaxique compte, chaque mot devient fondamental, et le résultat final est d'une justesse incomparable.

\subsection{Langage, réalité et intensification}

Les procédés stylistiques liés à l'intensification, que nous avons identifiés en grande quantité dans le texte "aident le lecteur à s'orienter, découvrant une romancière qui se réclame d'une transparence extrême" (Benali, 2008: 28). Amélie Nothomb est transparente comme l'eau, son élément préféré et adoré: “L'eau en dessous de moi, l'eau au-dessus de moi, l'eau en moi - l'eau, c'était moi. Ce n'était pas pour rien que mon prénom, en japonais, comportait la pluie." (Nothomb, 2000: 109). Ce n'est donc pas par hasard qu'elle a choisi Amélie comme prénom, sinon parce qu'il répond à la nécessité de concevoir une nouvelle identité par le biais de la parole: ici plus que jamais, nommer, c'est donner la vie.

Dans notre recherche, nous avons repéré les nombreuses et différentes formules syntaxiques dont l'auteure se sert pour extérioriser l'intensité de ses émotions. Ceci nous permet de saisir la relation entre l'état intense et le besoin typiquement nothombien de créer un contexte émotionnel le plus réel possible, où la véracité objective n'est pas un but, tandis que la crédibilité de la perception intime des évènements devient la colonne portante du récit.

Nothomb a souffert depuis toute petite la conscience du départ, la répétition de l'abandon. Sa famille est toujours restée très unie, mais les voyages et déplacements liés aux nombreux changements d'affectation de son père ont marqué une enfance singulière, pour une fillette qui s'attachait facilement à des personnes qui ne partageaient que de courtes périodes de sa vie. En même temps, elle a vite compris la valeur du souvenir, dont le pouvoir de recréer une réalité perdue l'a séduite, ainsi que celui de féconder l'écriture pour qu'elle devienne

19 Cf. notamment: Collington T. (2013), "La 'scène' de l'ironie chez Amélie Nothomb" in L'ironie: formes et enjeux d'une écriture contemporaine, 77-98. Classiques Garnier.

20 Affirmation de l'auteure dans notre correspondance privée. 
créatrice. Le passage suivant, d'une beauté et d'une tendresse exquises, rend hommage à celle qui sut déclencher la puissance de la singularité chez un Dieu qui ne se déclinait qu'à la troisième personne.

Au moins mon souvenir lui conserve-t-il l'existence. Ma grand-mère avait essuyé les plâtres de ma mémoire. Juste retour des choses: elle y est encore bien vivante, précédée de son bâton de chocolat comme d'un sceptre. C'est ma façon de lui rendre ce qu'elle m'a donné. (Nothomb, 2000: 47).

Dans sa lutte contre l'oubli, l'écrivaine déploie tout son arsenal syntaxique, consciente du fait que l'écriture est un moyen privilégié pour communiquer la façon dont on comprend la vie. Nous avons identifié dans ce paragraphe un syntagme qui, agissant comme expression de l'intensité, produit un effet de renvoi à un espace narratif où le lecteur retrouve la réalité intime de l'auteure. Voyons la spécificité de l'expression "essuyer les plâtres" dans le contexte de l'histoire de Métaphysique des tubes. Le signifié littéral est transparent: c'est habiter pour la première fois une maison qui vient d'être construite, une habitation récemment achevée. Il en découle que celui qui occupe les lieux alors que les plâtres sont encore humides souffre des désagréments physiques clairs. Mais cette locution possède aussi un signifié métaphorique très intéressant pour notre étude: "subir le premier les conséquences d'une situation fâcheuse"21. D'abord, cela nous renvoie au passage relatif au moment où la grand-mère a su traiter, première dans la famille, la petite bête furieuse qui n'arrêtait pas de crier - une situation absolument fâcheuse puisque l'enfant se montrait véritablement fâchée contre tout le monde. De plus, l'écrivaine avait partagé auparavant dans le récit ${ }^{22}$ cette sensation d'ouverture et découverte subite de la mémoire que l'évènement du chocolat a suscité chez elle (dès lors, elle affirme se souvenir de tout ce qui se passe dans sa vie), en lui montrant la dimension du souvenir, qu'elle cherche toujours à valoriser dans ses textes. Finalement, grâce à ce jeu de renvois narratifs, une double couche métaphorique apparait dans cette expression; puisque les plâtres ne sont pas ceux d'une habitation quelconque, sinon les murs de la mémoire nothombienne, que l'intensité linguistique dessine comme un espace clos, une chambre fermée que rien ni personne n'avait encore réussi à pénétrer. L'image des plâtres est d'autant plus aigüe qu'elle nous fait penser à quelqu'un qui serait immobilisé dans un plâtre médical de contention, tel que cette enfant bizarre qui n'avait pas esquissé le moindre mouvement pendant les deux premières années de sa vie.

Face à un évènement aussi intense que la mort d'un être cher, l'écriture doit se montrer à la hauteur, elle doit atteindre un degré exagérément élevé afin de savoir rendre au souvenir son épaisseur de réalité vécue. Nothomb ne vise pas à produire des phrases 'crédibles' du point de vue de la véracité des faits et des témoignages, car ce qui l'intéresse est

21 Le Petit Robert (1990), fig. et fam. par métaph.

22 (Nothomb 2000: 35). 
plutôt la réalité des sentiments, la précision du souvenir intime, "de ce qui en valait la peine" (Nothomb, 2000: 35), c'est-à-dire: l'univers naturel entourant la petite fille au Japon, les quelques personnes belges ou japonaises qui font partie de sa vie et les aventures sensorielles vouées à la beauté, qui vont marquer son approche à la vie autant que son style d'écriture.

La personnalité de Nothomb s'est forgée lors de ses premières années japonaises, et c'est aussi un trait particulièrement japonais celui de vouloir ressusciter un passé magnifique, un âge doré ou tout était parfait, où le terrestre touchait le divin et s'en appropriait. Dans l'histoire de Métaphysique des tubes, cette prise de position est incarnée par la gouvernante Kashima-San, toujours prête à regretter la période précédant la Deuxième Guerre Mondiale, quand le Japon était encore un grand Empire et que ses habitants le considéraient "le plus beau pays du monde" (Nothomb, 2000: 119).

\subsection{Le mot comme sauveteur de l'identité}

Une coïncidence de choix stylistiques se révèle assez claire entre Amélie Nothomb et le poète espagnol prix Nobel de littérature Juan Ramon Jiménez; les deux sont extrêmement attentifs à la musicalité du discours, et le chemin qu'ils suivent vers la conquête d'un langage personnel passe par la musique intime ${ }^{23}$, ainsi que par la conviction qu'il faut rechercher un plan supérieur: d'une part, ce serait le rébus qui hante Nothomb; de l'autre, la persécution de quelque chose « detrás del horizonte, hacia lo que no se ve" (cité par Javier Blasco, cf. bibliographie) à laquelle s'adonne l'Espagnol durant toute la deuxième phase de sa production littéraire.

"Intelijencia, dame el nombre esacto de las cosas!"24: c'est une véritable prière que le poète andalou livre à son propre esprit, un souhait intense qui rappelle de près l'exclamation que la petite Amélie prononce avec désespoir, après avoir être informée par sa mère qu'un jour elle devrait obligatoirement quitter le Japon à cause du travail de son papa: "Tu dois te souvenir, tu dois te souvenir!" (Nothomb, 2000: 127).

Elle avait compris que seule la parole exacte pourrait garder dans sa vie autant sa patrie adorée que toutes ses merveilleuses expériences d'enfance.

L'importance de la littérature, de l'écriture et, plus généralement, de la langue dans la vie privée de Nothomb a été étudiée par de nombreux critiques, au point d'être véritablement morcelée dans de nombreuses publications et mémoires; qu'il nous suffise ici, pour la signaler en guise de résumé, la déclaration que l'auteure même a faite lors de la master class organisée en 2017 par France Culture: "Quand on perd tout, on garde les mots.” (Serrell, 2017: 107).

Le mot, grâce à sa fonction lyrique, est devenu sauveteur: en figeant dans un présent

$23 C f$. la très complète édition critique de Antología poètica de J. R. Jiménez prologuée et commentée par Javier Blasco, qui insiste sur l'importance de l'esthétique musicale d'une poésie profondément sensible et recherchée.

24 Juan Ramon Jiménez, Antología poética, 287. 
éternel l'identité de l'auteur et par conséquent son monde, tel qu'un abri littéraire, il offre au je une protection du néant, de l'oubli et finalement de la disparition. Nothomb suit ce même procédé par rapport à son enfance, qu'elle désire intensément garder intacte dans la mémoire, afin de lui éviter une mort qui serait insupportable à ses yeux: "La seule vraie mort est l'oubli" (Nothomb, 1992: 182) c'est ce qu'affirme aussi le vieil écrivain cynique Prétextat Tach, protagoniste d'Hygiène de l'assassin (œuvre de début de Nothomb), dans un passage quasi-philosophique où il explique à une journaliste les raisons apparemment incompréhensibles qui l'ont mené à tuer sa jeune cousine Léopoldine.

Ces deux magnifiques écrivains, Nothomb et Juan Ramón, professent une foi sans bornes en la parole créatrice. Ce pouvoir les soutient dans leur recherche infatigable de la perfection syntaxique: si l'Espagnol fonde ses dernières œuvres poétiques sur l'exigence personnelle d'atteindre la plus grande correspondance entre la réalité expérimentée et celle qui est reconstituée par les mots ${ }^{25}$, la réalité créée par Amélie Nothomb grâce à l'écriture se superpose directement à celle qui a été vécue. C'est la liaison virtuelle qui unit autobiographie et fiction, et qui la pousse à glisser vers l'autofiction:

Podemos concluir que el yo, al dirigir su atención hacia si mismo, convierte la escritura en un instrumento de autoindagación. Escribe también para dejar un rastro, un signo que le proporcione la certeza de que aquello que vivió fue real. (Durá Celma, 2010: 174).

Nous avons vu comment la parole est capable d'échafauder la structure des souvenirs afin de bâtir une existence effective qui devient indiscutable ; nommer est donc une opération du langage qui consiste à construire un concept à travers une forme, en combinaison avec d'autres signes.

Amélie Nothomb ne s'arrête pas à ce premier niveau. Par le procédé de la nomination, elle veut construire non seulement un concept, mais un contexte de nature solide et factuelle, peu importe qu'il soit aussi véritable, puisque la vérité relève des sentiments et des sensations, beaucoup plus souvent que des faits.

Face à un univers informe et insensé, l'écrivain est contraint à jouer les démiurges. Sans l'agencement formidable de sa plume, le monde n'aurait jamais été capable de donner des contours aux choses, et les histoires des hommes auraient toujours bée comme d'effarantes auberges espagnoles. (Nothomb, 1992: 178).

Nommer, c'est donner forme aux êtres. La classe conceptuelle des êtres est caractérisée par son indépendance sémantique (Charaudeau, 1992: 47), c'est-à-dire que les êtres ne se définissent pas par rapport à une référence externe, mais leur définition de sens est inhérente

25 Dans la très connue composition qui ouvre la collection intitulée Eternidades, le poète s'interroge avec une énorme intensité sur la possibilité de donner vie au nom exact des choses, celui qui correspond à la réalité puisqu'il la crée, la retrouve en soi et lui donne sa vraie forme. 
à eux-mêmes. Amélie Nothomb applique souvent une technique de création linguistique qui consiste à définir un être à partir de morphèmes appartenant à d'autres classes conceptuelles, qui au départ ne sont pas sémantiquement indépendantes: l'exemple du verbe être est ici à notre avis le plus éclatant.

Dans la première page de Métaphysique des tubes, il s'agit de la genèse, du big bang personnel de l'auteure: c'est la naissance de Dieu qui nous est racontée dans cet incipit tout à fait particulier ${ }^{26}$. Dans le cadre de l'analyse linguistique, nous avons remarqué la présence d'un protagoniste lexical plus qu'évident: le verbe 'être'. Ce verbe apparaît jusqu'à onze fois dans le premier sous-chapitre pour créer une montée sémantique dans l'échelle de la réalité, de l'existence tangible. Le sommet de ce crescendo de puissance sémantique prédicative ne pouvait être atteint que par une tautologie, encore une fois d'inspiration biblique ${ }^{27}$, telle que: "Dieu était Dieu" (Nothomb, 2000: 6). Comment arrive-t-on à oser écrire une telle expression?

Dans cet extrait d'une page et demie, avant d'atteindre une telle figure d'intensité, le verbe est décliné dans toutes ses variantes:

- dans une phrase négative dont la valeur sémantique est cependant positive: "Et ce rien n'était ni vide ni vague".

- $\quad$ suivi par des adjectifs qualificatifs qui ne contiennent pas le sens d'intensité, mais qui sont employés dans une comparaison de haut degré: "Il était plein et dense comme un œuf dur".

- dans une construction d'intensité, avec un adjectif qualitatif dont la valeur sémantique est du superlatif: "Dieu était l'absolue satisfaction".

- dans une construction d'intensification qui ajoute l'opposition à la comparaison: "La vie était à ce point plénitude, qu'elle n'était pas la vie". Nous observerons que cette construction se rapproche aussi du sophisme, vu que la logique reliant la prémisse à la conclusion est fallacieuse, puisque la vie ne peut pas être autre chose que la vie même.

- comme caractéristique intrinsèque, dans une définition sémantique qui relève de l'axiome: "Dieu était satiété et éternité"28.

\subsection{Accroître l'intensité par les temps verbaux}

Nothomb considère l'incipit comme une pièce fondamentale dans l'objectif d'un bon développement du roman: elle a affirmé à plusieurs reprises que le début d'un texte doit atteindre une simplicité radicale, que l'écrivain devrait rencontrer par un souci de perfection qui l'amène à limer, polir et enlever tout ce qui pourrait être superflu.

26 Pour une étude approfondie de l'incipit de Métaphysique des tubes, cf. Benbassal (2015).

27 Moïses: "Je suis celui qui est".

28 Cet énoncé a été décrit plus en profondeur dans le chapitre 4 de cet article. 
Par rapport à la gestion des temps verbaux, elle manifeste aussi une volonté d'intensification qui dépend de la nécessité de construire la réalité. L'exemple le plus éclatant nous semble celui du passage où Nothomb relate l'accident de l'étang aux carpes (simple accident ou possible tentative de suicide?). Soudain, elle passe au présent, tandis que, depuis le début, le livre avait été écrit au passé, le plus souvent à l'imparfait: pourquoi ce changement imprévu? Nous avons affaire à ce que Charaudeau appelle du présent actuel (Charaudeau, 1992: 459), où le processus (l'action exprimée par le verbe) se trouve dans l'actualité du sujet parlant. C'est une façon de transmettre l'intensité, car c'est comme si la narratrice redevenait petite pour communiquer son expérience du point de vue de l'enfant de trois ans: "O eu-adulto existe ao serviço do eu-criança, buscando retratar suas experiências infantis" (Azzi Ferreira, 2007: 16). Pour ceci, elle a besoin de créer un effet d'immédiateté que l'imparfait ne sait pas susciter, puisqu'il se situe dans une portion de transposition du présent (Charaudeau, 1992: 462) où le processus décrit est antérieur au passé, mais demeure lié à celui-ci.

Dans le cadre de notre étude, le rapport entre le passage écrit au présent et le reste du livre souligne la gravité de l'évènement. Ce moment de sa vie possède la force d'un moment fondateur de l'existence de l'auteure. Le présent actuel a la fonction d'intensifier l'étendue du moment et de le rendre ainsi éternel: "cela fait une éternité que je suis entre deux eaux." (Nothomb, 2000: 149).

Un autre passage essentiel dans la création de la réalité à travers l'expression de l'intensité est probablement celui de la transformation du tube en enfant, grâce à la découverte du plaisir, incarné par un bâton de chocolat blanc (belge! ${ }^{29}$ ). Du point de vue lexical, ces deux pages représentent une véritable explosion d'intensité: Nothomb emploie plusieurs procédés, tels que la répétition, les références bibliques, les points d'exclamation, l'intensification adjectivale, l'opposition... mais il y a un élément linguistique particulier qui marque la différence par rapport aux premières pages du récit: c'est le passage de la troisième à la première personne du singulier, qui se produit simultanément à la transformation physiologique qui opère chez le tube. C'est le moment qui pointe le début de la perspective personnelle d'Amélie sur le monde qui l'entoure et qui commence par la découverte de la verbalité, "outil indispensable dans la quête des plaisirs" (Szucs, 2007: 83).

La projection de la première personne du singulier sur l'identité de la protagoniste possède une force créatrice tellement notable que le procédé contraire révèle une capacité destructrice aussi forte: quand Amélie tombe (se laisse tomber) dans l'étang, elle sent petit à petit que le tube reprend possession de son corps et de son esprit, et cette néantisation (Álvares, 2012: 257) est verbalisée par le retour à la troisième personne.

Nothomb met en place ce que Charaudeau (1992: 85) appelle le "principe de l'expression de son identité à travers le langage.” Le langage devient en même temps sujet et objet

29 Ce n'est pas la langue nationale qui fait émerger le moi, mais le plaisir national du chocolat. (Álvares, 2012: 249). 
de son discours, et le lecteur accomplit un rôle essentiel dans la construction du discours, puisqu'il accompagne l'auteure sur le chemin de l'introspection, sans plus se questionner sur la véridicité du récit, grâce à l'effet de recréation du contexte obtenu par le biais de l'intensification.

\section{Conclusion}

Nous avons commencé notre étude par un aperçu du style narratif très particulier de l'écrivaine belge. Le concept d'autofiction nous a aidé à mettre en lumière certains aspects de l'écriture nothombienne qui auraient pu entraîner une certaine confusion; notamment, dans Métaphysique des tubes, le mélange de faits vrais et de passages fabuleux.

Notre but était de déceler la relation que Nothomb établit entre la perception de la réalité et son explicitation à travers les ressources linguistiques de l'intensité. Nous avons souligné jusqu'à quel point l'emploi de l'intensif révèle la présence explicite du locuteur, qui est le responsable évident du choix de l'expression signalant l'intensité, ainsi que celui qui éprouve en première personne le degré émotionnel qui entoure la réalité perçue et exprimée comme intense.

Nous avons aussi souligné que Métaphysique des tubes est un livre très dense, beaucoup plus complexe que ce qu'une première lecture pourrait révéler. La syntaxe est profondément travaillée et chaque lexie employée possède une fonction précise dans l'économie linguistique du texte, où le langage devient en même temps le sujet et l'objet de l'écriture, dans une sorte de mise en abîme qui renvoie non seulement à la production littéraire, mais, de façon plus ambitieuse, à une philosophie de l'apprentissage valable pour tous les êtres humains.

Le texte est lui-même structuré comme un tube: un trou noir qui contient tout et qui attire tout ce qui l'entoure en provoquant une explosion d'énergie autour, l'énergie infatigable qui accompagne la petite Amélie pendant ses trois premières années de vie. Nous avons repéré un mot qui traverse tout le récit de façon subtile, mais incisive: c'est le mot rien, qui revient jusqu'à 39 fois dans le livre, mais qui se trouve surtout dans deux énoncés mémorables de la première et la dernière page: “Au commencement il n'y avait rien." (Nothomb, 2000: 5) et "Ensuite, il ne s'est plus rien passé.” (Nothomb, 2000: 157). Grâce à cet expédient linguistique, c'est comme si l'univers entier s'écoulait sous les yeux du lecteur en trois ans: il commence par un big bang et se termine sur un trou noir, mais entre les deux évènements, tout n'est qu'intensité.

Tandis que la phrase d'ouverture du texte est une affirmation descriptive qui relève de la Bible, l'énoncé final contient une magnifique hyperbole, par laquelle l'auteure renouvelle le jeu qu'elle entretient depuis le début avec ses lecteurs: son message exagéré est capable de créer ou de détruire à travers le langage, et c'est le récepteur qui est censé en récupérer 
MÉTAPHYSIQUE DES TUBES D'AMÉLIE NOTHOMB: UNE VIE RACONTÉE À TRAVERS L’INTENSITÉ...

la vérité, parce que: "lire ne constitue pas seulement une situation d'apprentissage, mais de passage à l'acte.” (Amanieux, 2005: 109).

Nothomb trompe volontairement son public. Bien sûr, ce n'est pas vrai qu'après le troisième anniversaire il ne s'est plus rien passé dans sa vie: bien de sensations concentrées et d'émotions inoubliables (inoubliées) ont rempli ses jours, et le récit de toutes ces émotions a rempli de nombreux livres depuis lors.

Ce qui s'est passé surtout, c'est qu'elle a découvert que le langage, l'expression écrite et la littérature permettent de vivre la vraie vie, celle des souvenirs à la manière de Proust, des émotions incarnées par les personnages - selon la tradition classique-, du procès d'écriture comme jouissance et plaisir. Pour paraphraser à l'envers cette clôture catégorique, nous affirmons très intensément qu'ensuite il s'est passé toute une vie.

\section{Références bibliographiques}

Noтномв, Amélie (1992). Hygiène de l'assassin. Paris, Albin Michel.

Noтномв, Amélie (2000). Métaphysique des tubes. Paris, Albin Michel.

Álvares, Cristina. 2012. "Une subjectivation extravagante. Métaphysique des tubes, d'Amélie Nothomb" in Carnets IV, (Res)sources de l'extravagance, 245-259.

Amanieux, Laureline. 2005. Amélie Nothomb: l'éternelle affamée. Paris, Albin Michel.

Azzi Ferreira, Christine. 2007. "Je suis un autre': Amélie Nothomb e a escrita autobiográfica" in Palimpsesto, V. 7, 1-20.

Benali, Souâd (2008). Fonctionnement du discours humoristique dans le texte littéraire. Le cas de l'œuvre romanesque d'Amélie Nothomb, Thèse doctorale, Université d'Oran. Algérie.

Benbassal, Khadidja (2015). "Étude de l'incipit et de la clôture dans 'Métaphysique des tubes' et 'Antéchrista' d'Amélie Nothomb, Mémoire de Magistère en sciences du langage, Université d'Oran 2, Algérie.

Charaudeau, Patrick (1992). Grammaire du sens et de l'expression. Paris, Hachette Éducation.

Colonna, Vincent (1989). L'autofiction: essai sur la fictionnalisation du soi en littérature. Paris, Mémoire, École des Hautes Études en Sciences Sociales.

Delangue, Henri. 2014. “Autobiographie ou autofiction chez Amélie Nothomb?” in Çédille, Revista de estudios franceses, $\mathrm{n}^{\circ} 10,74-89$.

Do Nascimento, Takiko. 2008. "La comparaison dans Biographie de la faim d'Amélie Nothomb" in Lettres françaises, $n^{\circ}$ 9, Universidade Federal da Bahia. Instituto de Letras Departamento de Letras Románicas, 75-83.

Durá Celma, Rosa. 2010. “En el principio...fue el deseo. La constitución del yo en Metafi- 
sica de los tubos: una lectura desde la teoría psicoanalítica" in Cuadernos de investigación Filológica, no 35-36, 165-183.

JimÉnEz, Juan Ramón. 2008. Antología poética. Edición de Javier Blasco. Madrid, Cátedra.

F. LÁzARo, Fernando \& Vicente Tusón. 1990. Literatura española, Madrid, Anaya.

LeJeune, Philippe. 2005. Le Pacte autobiographique, Paris, Éditions du Seuil.

Polguere, Alain. 2002. "Modélisation des liens lexicaux au moyen des fonctions lexicales" in Actes de la 9ème conférence sur le Traitement Automatique des Langues Naturelles. Nancy, Association pour le traitement automatique des langues, Nancy, 37-60.

Romero, Clara. 2007. "Pour une définition générale de l'intensité dans le langage" in Travaux de linguistique, col. 54, $\mathrm{n}^{\circ} 1,57-68$.

Serrell, Mathilde. 2017. "Je vis une dictature de l'écriture absolue" in Papiers, Revue de France-culture, $n^{\circ} 23,106-113$.

Szucs, Ágota. 2007. «Moi et la Toupie». Analyse de Métaphysique des tubes d'Amélie Nothomb, in Acta Romanica Szegediensis, Tomus XXV.

Zumkir, Michel. 2007. Amélie Nothomb de A à Z, Portrait d'un monstre littéraire. Paris, Grand Miroir. 
